\title{
Smoothening the Lid-Cheek Junction in Lower Blepharoplasty
}

\author{
MOHAMED ASHRAF, M.D.; AYMAN NOAMAN, M.D. and AHMED ELNAGGAR, M.D. \\ The Department of Plastic \& Reconstructive Surgery, Faculty of Medicine, Cairo University, Egypt
}

\begin{abstract}
Aim: Transcutaneous lower blepharoplasty is more convenient for its easier learning curve, its better visibility and access to the lower periorbital structures. Conventional techniques entail fat removal to improve the lower eyelid contour. In this study we adopted the extended transcutaneous lower eyelid blepharoplasty that included dissection below the extent of the inferior orbital margin to correct the tear trough deformity that is usually missed in traditional techniques.

Methods: From December 2017 to December 2018, 25 patients were subjected to this procedure via a transcutaneous stair-step incision. A retrospective analysis was implemented. On the medial side, palpebral, orbital orbicularis oculi muscle and the orbitomalar ligament were freed with dissection approaching to the premaxillary space. On the lateral side, release of the orbitomalar ligament was done, followed by dissection reaching to the prezygomatic space. Fat pads were dealt with either by excision or redistribution.
\end{abstract}

Results: The mean follow-up was 12 months. High degree of patient's satisfaction was noted after using this technique. No major complications in term of lid malposition or scleral show or infection.

Conclusion: Extended transcutaneous lower blepharoplasty is a safe and effective approach and a very powerful modality for blending the lid-cheek junction and periorbital rejuvenation.

Level of Evidence: Therapeutic, V.

Key Words: Blepharoplasty - Orbitomalar ligament - Orbicularis retaining ligament - Periorbital rejuvenation.

\section{INTRODUCTION}

The main objective of lower blepharoplasty is to regain the youthful appearance of the lower eyelid, and this usually means a smoother eyelidcheek junction [1].

The anatomy of the groove lying between the lower eyelid and the upper cheek has been analyzed precisely in the last few years. From the inner aspect, this groove coincides with the attachment of the orbicularis muscle, and in its outer and central portion it corresponds with the insertion of the orbicularis retaining ligament $[2,3]$.

Conventional lower blepharoplasty generally focuses on excision of skin, muscle, and fat which might be cosmetically beneficial in some patients, however, emergence of many possible remote complications with this procedure may be elicited including lower lid retraction, scleral show, distortion of the palpebral fissures, and hollowness of the lower eyelid region [4]. In the other hand, modern lower blepharoplasty tends for more conservative fat and skin resection or fat repositioning with negligible skin excision along with lower lid lifting via either canthopexy or canthoplasty procedures $[5,6]$.

Periorbital rejuvenation was extremely advanced. Thanks to the role of brow and midface surgeries, indicating the great importance of comprehensive facial assessment for optimal results [7].

Orbicularis retaining ligament release is usually essential for muscle mobilization and skin redraping. Lysis of the orbicularis retaining ligament, medial orbicularis oculi and the tear trough ligament has become a fundamental step in most lower blepharoplasties used to treat the lid-cheek junction deformities. Orbicularis retaining ligament is an osteo-cutaneous structure extending from the inferior orbital margin to the dermis of the lid-cheek junction passing through orbicularis muscle. Repair of the tear trough deformity usually necessitates lysis of the medial orbicularis oculi and orbicularis ligament. The end point of dissection can be achieved when the levator labii superioris muscle was seen clearly. This ligament spins in a circular pattern along the orbital margin, constituting the upper boundary of the preseptal space in the upper eyelid and the inferior aspect of the preseptal space in the lower eyelid [7,8] (Fig. 1). 


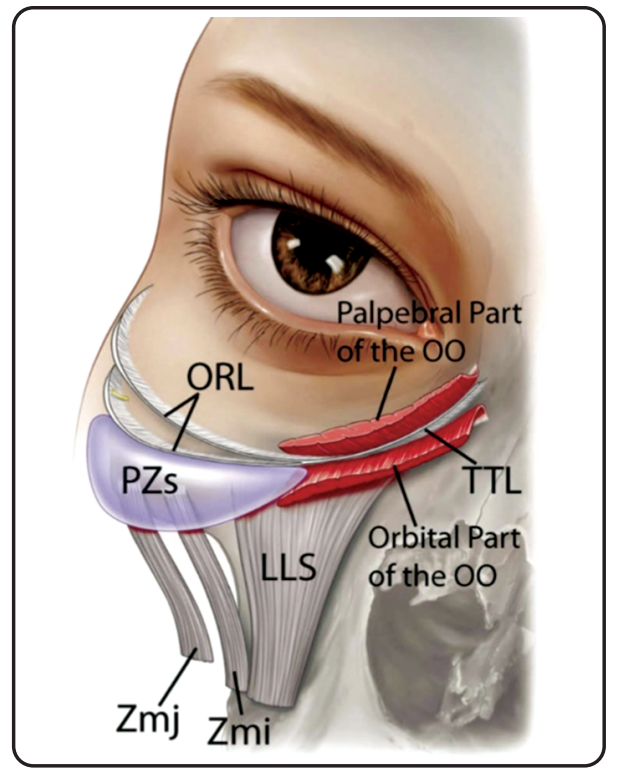

Fig. (1): Anatomy of the orbiculris retaining ligament and tear trough ligament. ORL, orbicularis retaining ligament. TTL, tear trough ligament. OO, orbicularis oculi muscle. PZs, prezygomatic space. Zmj, zygomaticus major muscle. Zmi, zygomaticus minor muscle. LLS, levator labii superioris muscle. "Quoted fromWong $\mathrm{CH}$, et al. [9]".

The orbicularis retaining ligament is short medially and laterally but longer in the middle (10 to $14 \mathrm{~mm})$. Accordingly, the central laxity of the orbicularis retaining ligament forms a V-shaped pattern [7].

Many patients have an obvious groove that makes the lower lid bags more prominent and exaggerates the lid-cheek junction. This periorbital depression corresponds with the meeting of orbicularis muscle, the infraorbital margin, and the orbicularis ligament. The part of that groove from the inner canthus to the midpupillary line is termed as the nasojugal groove, whereas the lateral portion is more frequently termed the "palpebromalar groove" [10]. The caudal extension of that groove appears characterizing a cheek bulge, however, the exact pathophysiology of that extension is still obscure [11].

\section{PATIENTS AND METHODS}

The authors performed a retrospective analysis of 25 patients in whom extended lower lid blepharoplasty was executed using the transcutaneous approach. Their age ranged from 37 to 52 years. All patients were nonsmokers. Patients with negative vector lids and those with premorbid orbicular weakness were excluded from the study. Close follow-up of the patients were completed over a period between 6 to 12 months.
The procedure was performed while the patient receiving IV sedation. Local anesthesia was infiltrated including lidocaine $2 \%$ with epinephrine $1: 150,000$.

Infiltration was done to the fat pockets and the suborbicularis plane in the whole lower lid down to $2 \mathrm{~cm}$ below the orbital margin.

A subciliary incision $2 \mathrm{~mm}$ from lash line was performed and then a myocutaneous flap (skinmuscle flap) was elevated after preserving a strip of $5 \mathrm{~mm}$ of the pretarsal orbicularis 5,6. Muscle incision was done to enter the preseptal plane. Lysis of the medial orbicularis muscle was performed followed by release of the orbicularis ligament till the medial corneal limbus. (Fig. 2).

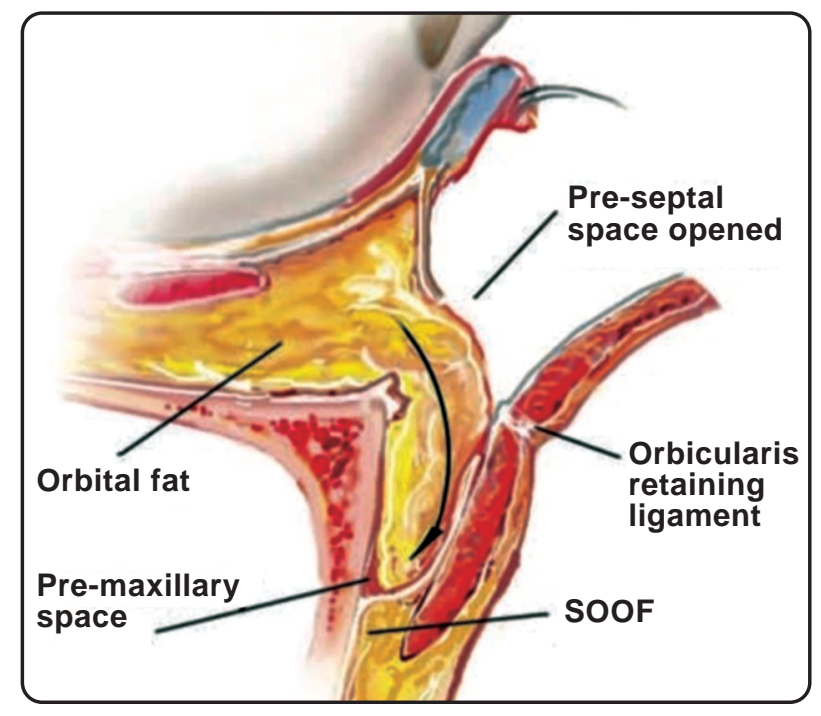

Fig. (2): Transcutaneous approach along with lysis of the orbicularis retaining ligament and mobilization of the sub-septal fat over the orbital margin beneath the muscle-skin flap approaching to the SOOF (sub orbicularis oculi fat) to obliterate the nasojugal groove."Quoted from Alghoul M. [12]".

Adequate dissection over the inferior orbital rim was performed until the levator labii superioris was clearly seen. This step indicates adequate interruption of the tear-trough ligament. The lysis proceeds till the level of the nasojugal / palpebromalar groove detected pre-operatively.

Supraperiosteal dissection was chosen, because the periosteum endues minimal lifting and additionally, the vascularity of the mobilized fat flap was preserved. Release of the inferior aspect of the orbital septum was performed followed by fat transposition over the orbital rim. Fixation of the transposed fat can be achieved either to the skin or in situ. (Fig. 3). 
(A)

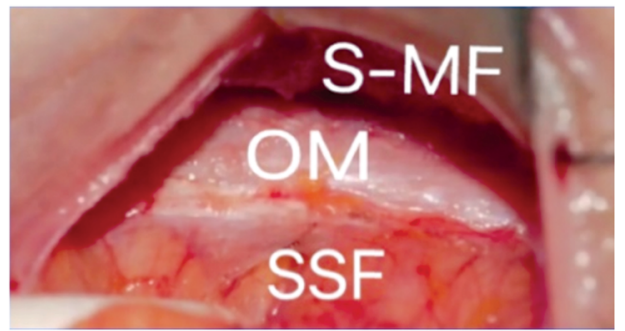

(C)

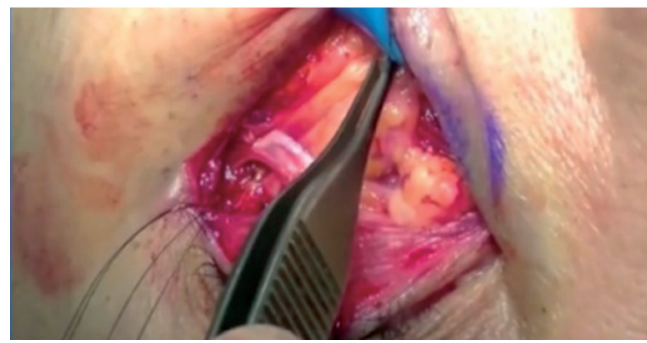

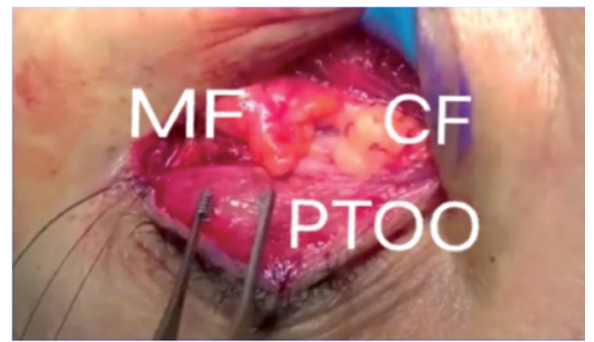

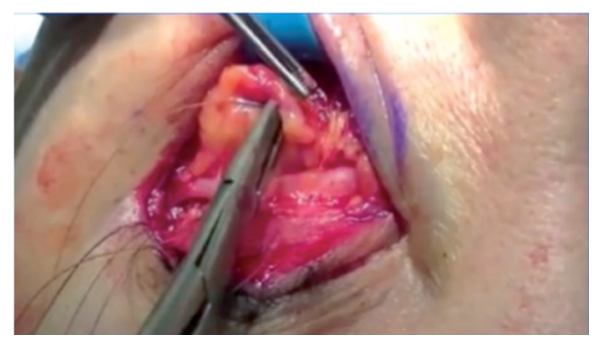

(D)

Fig. (3): (A) Elevation of the skin-muscle flap exposing the orbital margin after releasing the orbicularis retaining ligament, orbital septum and sub-septal fat. (B) The orbital septum opened showing the medial fat pad and central fat pad. (C) Mobilization of the central and medial fat pads as a pedicled flaps that can be advanced over the orbital margin. (D) The fat flaps were sutured beneath the muscle medially to correct the tear-trough deformity. SSF, sub-septal fat; S-MF, skin muscle flap; OM, orbital margin; CF, central fat pad; MF, medial fat pad; PTOO, pretarsal orbicularis oculi muscle.

Conservative fat excision was performed in 18 cases and transposition of the medial and central fat pockets in 7 cases with routine excision of the lateral fat pocket.

Manipulation of the sub-septal fat depended mainly on the severity and the degree of the tear trough deformity. Patients with mild to moderate deepening of the nasojugal fold required conservative fat excision only. On the other hand, fat transposition was reserved for patients with severe well demarcated hollowness.

Tightening of the canthal laxity was a fundamental step of that technique. In the other hand, lateral tarsal canthopexy was conducted if there was extensive lower eyelid laxity or lateral canthal tendon weakness, this method aimed at approximating the lateral tarsus to the lateral orbital margin using a 4-0 Prolene horizontal mattress suture.

Re-draping of the myocutaneous flap was achieved by fixing the orbicularis muscle to the periosteum of the lateral orbital margin with a supero-lateral vector with 5-0 PDS horizontal mattress suture followed by minimal skin excision of the superolateral part.

\section{RESULTS}

A total of 21 cases showed an obvious improvement with adequate blending of the lid-cheek junction with acceptable results and overall satisfaction to both patient and surgeon. (Figs. 4-9).
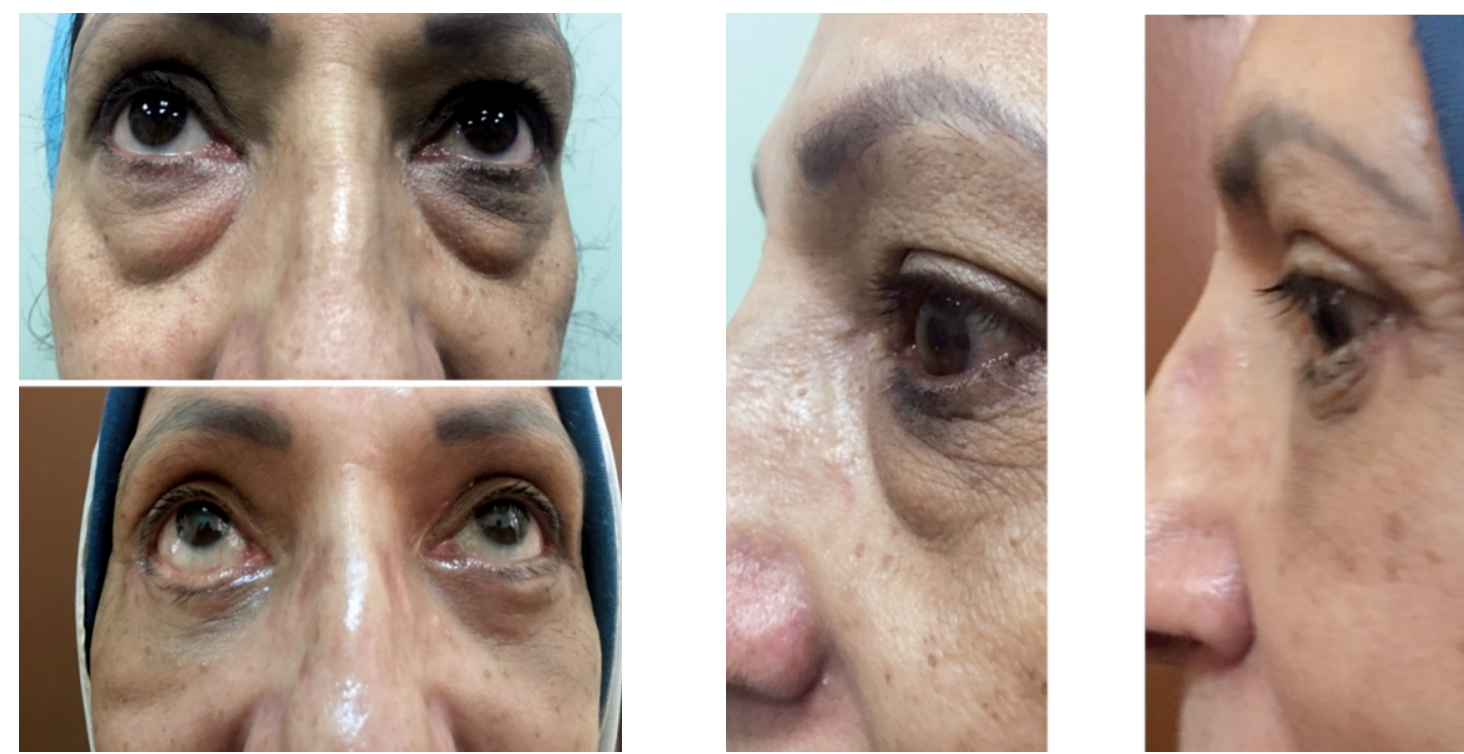

Fig. (4): Lower blepharoplasty with fat redistribution 6 months post-operatively. 

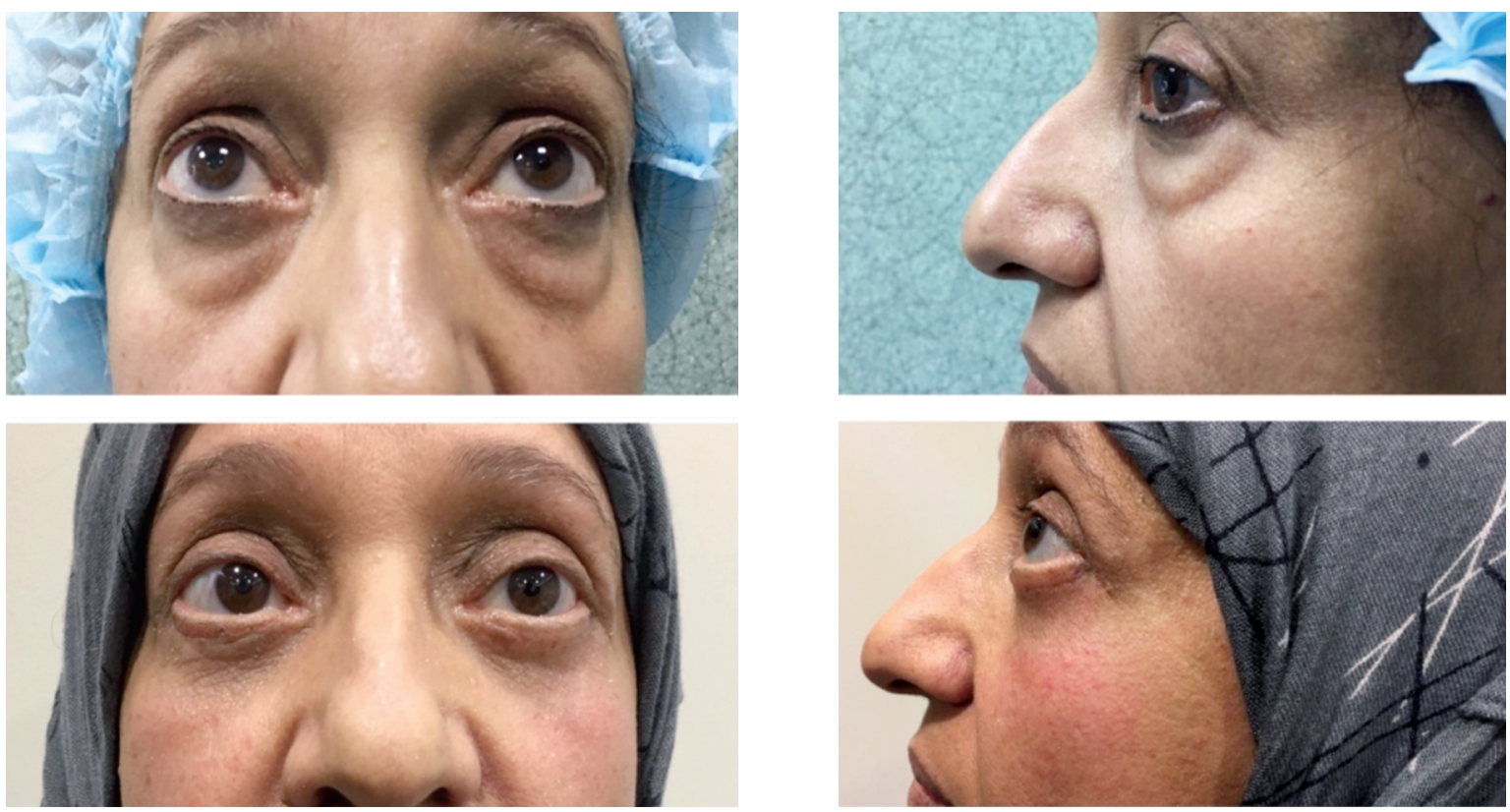

Fig. (5): Lower blepharoplasty with fat redistribution 3 months post-operatively.
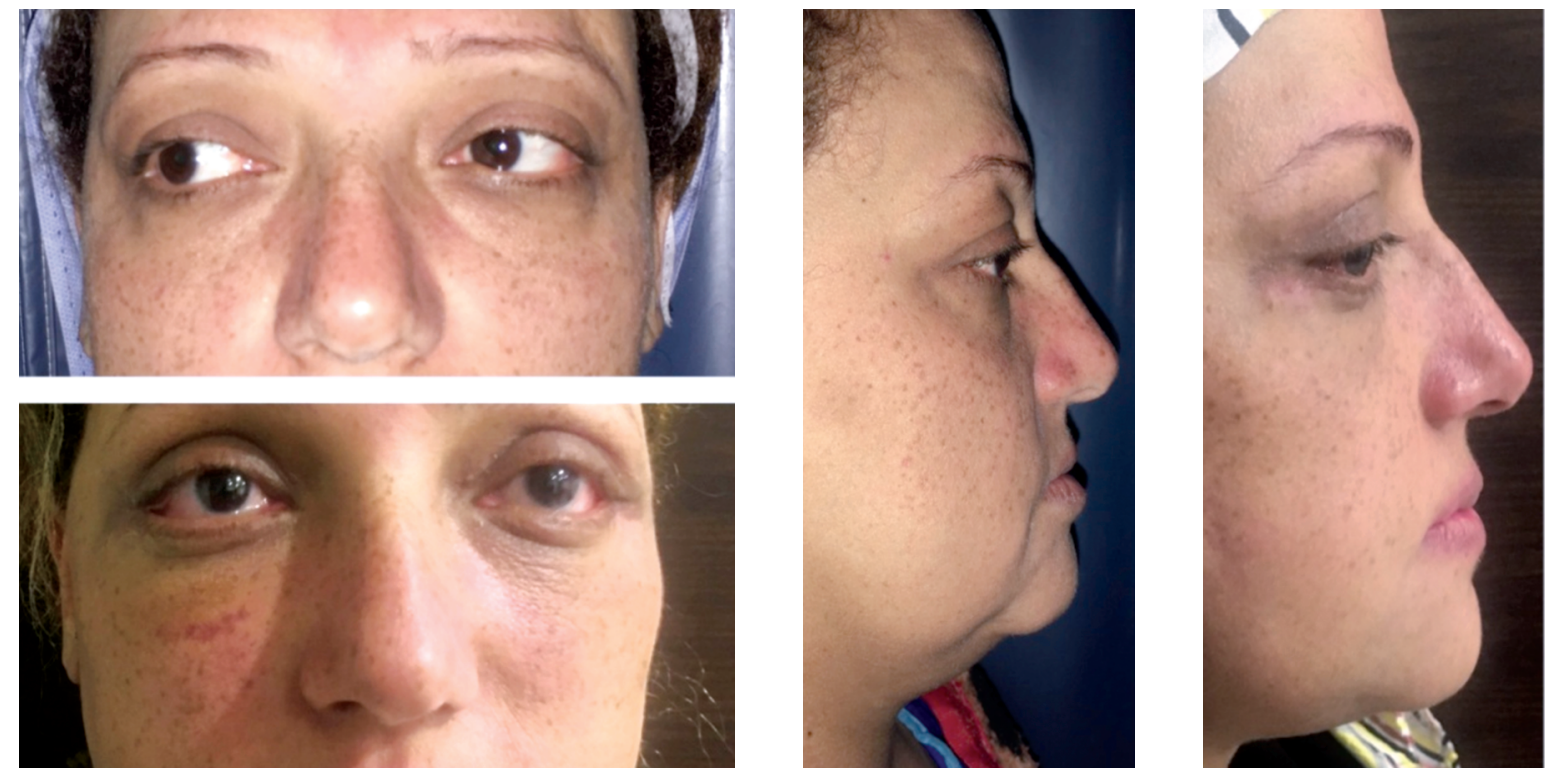

Fig. (6): Lower blepharoplasty with fat excision 3 months post-operatively.
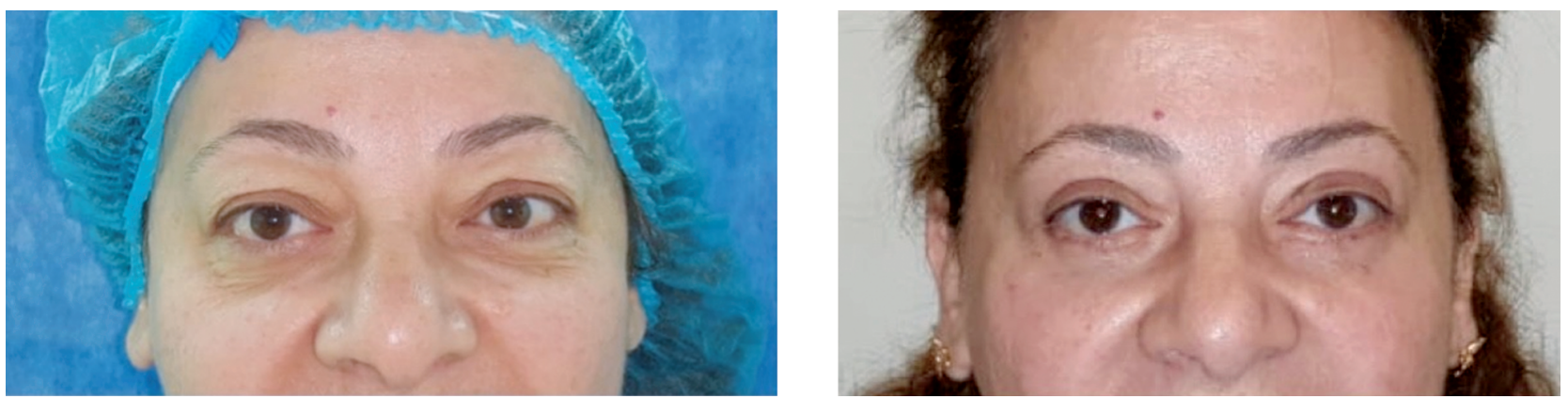

Fig. (7): Lower blepharoplasty with fat excision 12 months post-operatively. 

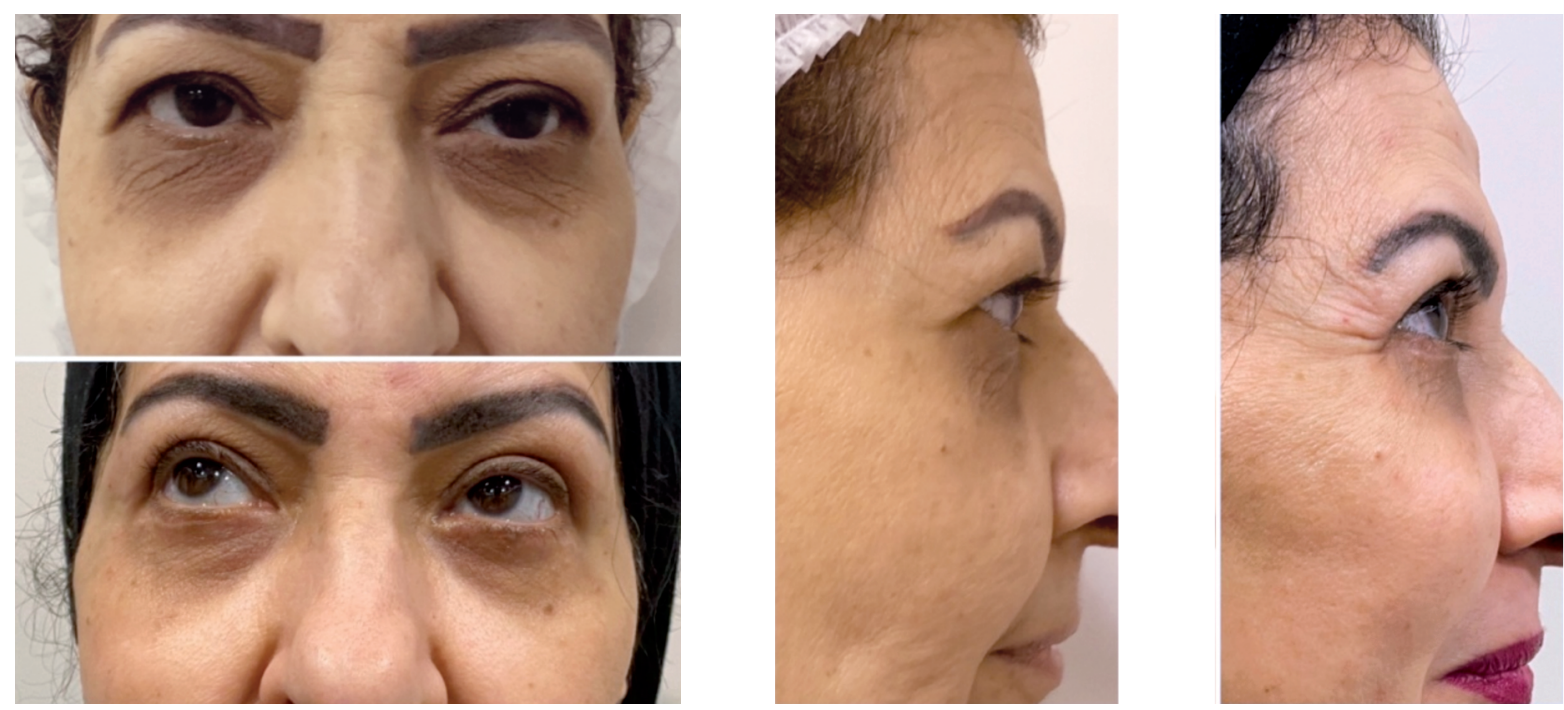

Fig. (8): Lower blepharoplasty with fat redistribution 12 months post-operatively.
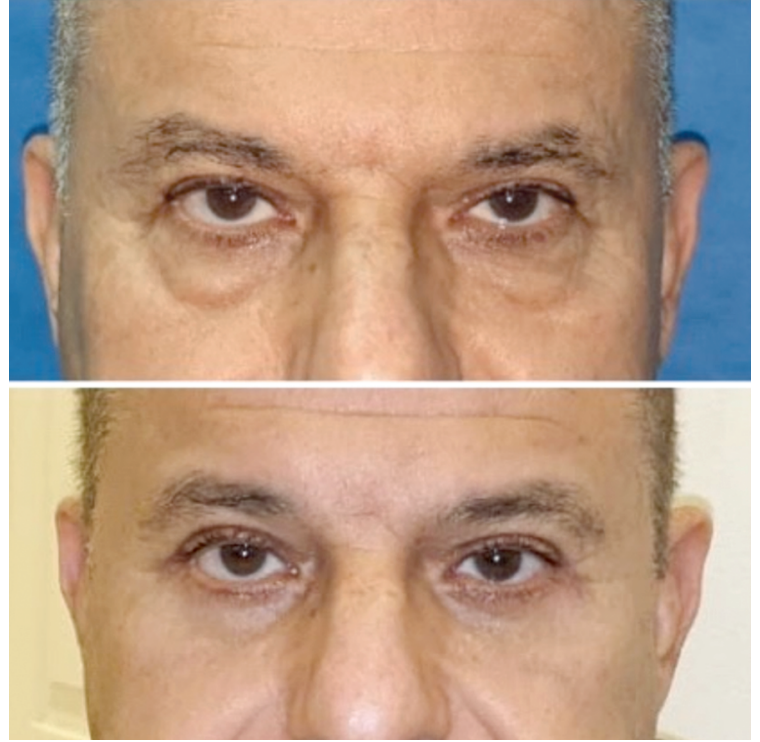

Fig. (9): Lower blepharoplasty with fat excision 12 months post-operatively.

Post-operatively, the results show adequate correction of the lid-cheek junction hollowness along with creation of an obvious youthful eyelid following manipulation of the sub-septal fat (either excision or transposition) coupled with sufficient release of the orbicularis retaining ligament. Additionally, the orientation of the lower lid margin and the severity (width) of the nasojugal groove were both improved.

Post-operative irregularities were noted in 4 patients who resolved over 3 months. The causes were either post-operative scarring or fat necrosis resulting from inadequate vascularity to the transposed fat. Fat necrosis was noticed in these patients throughout the follow-up period (subjectively) in a form of reduced size of the fullness (that resulted immediately post-operatively) in the nasojugal groove resulting in appearance of milder form of tear trough deformity.

Other post-operative adverse sequelae include chemosis which was reported in 14 out of the 25 patients with complete resolution within 2-3 weeks post-operatively. Neither lid malposition nor scleral show was noted.

\section{DISCUSSION}

Recent anatomical studies and understanding of the periorbital region, especially the surgical applications and the detailed classification of the fat compartments of the different parts of the face, formed the main principles for modern lower lid blepharoplasty. The precise evaluation of the facial fat compartments including the malar mound has a great role that enables the aesthetic surgeon to achieve the adequate balance at the lower lid-cheek junction $[13,15]$.

Although the orbitomalar groove is the cardinal deformity of the aging lower eyelid and midface, its repair has only lately become a focal point of lower blepharoplasty. Orbicularis suspension and re-draping has been known as an essential step in lid-cheek junction modification for the past three decades. Hamra was the first who combined both orbicularis and face lifting in the early 90's. He included 152 cases done over a 3-year period with acceptable outcomes and minimal drawbacks. He stated that despite the improvement that may happen in the appearance of the eye in traditional blepharoplasty, the outcomes are usually unexpected and hollowness at the lid-cheek junction may still there without any significant change. In the 
other hand, transposition of sub-septal fat along with adequate lysis of the orbicularis ligament will result in restoring the truly youthful eyelid [16].

Recently, McCord et al., have mentioned the improvement of midface contour afforded by orbicularis lifting in lower blepharoplasty [14-17].

A five-step lower blepharoplasty was conducted by Rohrich et al., who included blunt dissection of the orbicularis ligament altogether with upper cheek fat transfer. They included 50 patients in their study, and they achieved an $80 \%$ decrease in pupil-nasojugal fold distance along with a $79 \%$ diminution of the nasojugal fold width following lysis of the orbicularis ligament. Their results suggested that lysis of the tear trough ligament resulted in confluence of the sub-septal fat with the cheek fat resulting in a smoother lid-cheek junction [18].

Wong and Mendelson performed transconjunctival blepharoplasty with fat grafting in the nasojugal groove in 54 cases. They did adequate detachment of the orbicularis ligament medially and laterally along with fat transfer to obliterate the depression at the lid-cheek junction. They achieved satisfactory results with $2 \%$ revision rate [19].

Primary benefits from the technique we adopted are the possibility to tighten the lower eyelid adequately and the capability to maintain the preoperative eye shape. Dealing with the periorbital fat differs from case to another. The fat can be excised or redistributed to mask the tear-trough deformity. The adequate precise lysis of tethering usually lowers the incidence of downward traction on the lower lid, accordingly, lower the possibility of lower lid malposition, and thus obviates the step of extensive lateral canthal fixation.

Now, it is well known that optimum postoperative lower lid contour relies mainly on cheek lifting and stabilization combined with conservative skin excision and minimal canthal manipulation $[18,20]$.

Free fat transfer has its own drawbacks including fat resorption, necrosis, infection, later fat expansion or oil cyst formation. However, fat transposition is not feasible in cases of negligible periorbital fat. Lysis of orbicularis ligament in patients with mild lower lid redundancy is usually enough without a need of lateral canthopexy as the impact on the orbitomalar sulcus is direct and expected.

Complication rates of this method does not differ from other blepharoplasty techniques. How- ever, lower lid irregularities that happened in 4 patients were either due to lower lid scarring or partial necrosis of the transposed fat secondary to vascular impairment. The reduction of fat size resulted in reappearance of milder forms of tear trough deformity.

\section{Conclusion:}

Release of the orbicularis oculi along with release of the orbicularis ligament paves the way for the achievement of a smooth lid-cheek interface and lifting of the orbicularis and malar mound simultaneously. Also, it allows for adjustment of the lower eyelid skin and malar mound contour using a simple lifting sutures after skin resection.

This method is safe, versatile, and reliable for modification of profound aging features of the eyelid and upper cheek, with a lower rate of complications.

\section{Ethical approval:}

This study was reviewed by the ethical committee at the Faculty of Medicine, Cairo University.

\section{Declaration of conflicting interests:}

The authors declared no conflicts of interests.

\section{Funding:}

The authors did not receive any financial support.

\section{REFERENCES}

1- Carraway J.H.: Volume correction for nasojugal groove with blepharoplasty. Aesthetic Plast. Surg., 30: 101-109, 2010.

2- Kikkawa D.O., Lemke B.N. and Dortzbach R.K.: Relations of the superficial musculoaponeurotic system to the orbit and characterization of the orbitomalar ligament. Ophthal. Plast. Reconstr. Surg., 12: 77-88, 1996.

3- Haddock N.T., Saadeh P.B., Boutros S. and Thorne C.H.: The tear trough and lid/cheek junction: Anatomy and implications for surgical correction. Plast. Reconstr. Surg., 123: 1332-1340; discussion 1341-1342, 2009.

4- Maf T.R., Chang S. and Friedland J.A.: Traditional lower blepharoplasty: Is additional support necessary? A 30year review. Plast. Reconstr. Surg., 128: 265-273, 2011.

5- Hidalgo D.A.: An integrated approach to lower blepharoplasty. Plast. Reconstr. Surg., 127: 386-395, 2011.

6- Codner M.A., Wol I J.N. and Anzarut A.: Primary transcutaneous lower blepharoplasty with routine lateral canthal support: A comprehensive 10-year review. Plast. Reconstr. Surg., 121: 241-250, 2008.

7- Moss C.J., Mendelson B.C. and Taylor G.I.: Surgical anatomy of the ligamentous attachments in the temple and periorbital regions. Plast Reconstr Surg., 105: 14751490; discussion 1491, 2000. 
8- Ghavami A., Pessa J.E., Janis J., Khosla R., Reece E.M. and Rohrich R.J.: The orbicularis retaining ligament of the medial orbit: Closing the circle. Plast. Reconstr. Surg., 121: 994-1001, 2008.

9- Wong C.H., Hsieh M.K. and Mendelson B.: The tear trough ligament: anatomical basis for the tear trough deformity. Plast Reconstr Surg. Jun., 129 (6): 1392-402. doi: 10.1097/PRS.0b013e31824ecd77. PMID: 22634656, 2012.

10- Stutman R.L. and Codner M.A.: Tear trough deformity: Review of anatomy and treatment options. Aesthet. Surg. J., 32: 426-440, 2012.

11- Kpodzo D.S., Nahai F. and McCord C.D.: Malar mounds and festoons: Review of current management. Aesthet. Surg. J., 34: 235-248, 2014.

12- Alghoul M.: Blepharoplasty: Anatomy, Planning, Techniques, and Safety. Aesthetic Surg J. Jan 1; 39 (1): 1028. doi: 10.1093/asj/sjy034. PMID: 29474509, 2019.

13- Rohrich R.J. and Pessa J.E.: The fat compartments of the face: Anatomy and clinical implications for cosmetic surgery. Plast Reconstr Surg., 119: 2219-2227; discussion 2228-2231, 2007.

14- Rohrich R.J., Pessa J.E. and Ristow B.: The youthful cheek and the deep medial fat compartment. Plast. Reconstr. Surg., 121: 2107-2112, 2008.
15- Rohrich R.J., Arbique G.M., Wong C., Brown S. and Pessa J.E.: The anatomy of suborbicularis fat: Implications for periorbital rejuvenation. Plast. Reconstr. Surg., 124: 946$951,2009$.

16- Hamra S.T.: Repositioning the orbicularis oculi muscle in the composite rhytidectomy. Plast. Reconstr. Surg., 90: 14-22, 1992.

17- McCord C.D. Jr., Codner M.A. and Hester T.R.: Redraping the inferior orbicularis arc. Plast. Reconstr. Surg., 102: 2471-2479, 1998 .

18- Rohrich R.J., Ghavami A. and Mojallal A.: The five-step lower blepharoplasty: Blending the eyelid-cheek junction. Plast. Reconstr. Surg., 128: 775-783, 2011.

19- Wong C.H. and Mendelson B.C.: Reply: Extended transconjunctival lower eyelid blepharoplasty with release of the tear trough ligament and fat redistribution. Plast. Reconstr. Surg., 142 (2): 236e-237e, 2018.

20- Hester T.R. Jr., Codner M.A., McCord C.D., Nahai F. and Gianno-Poulos A.: Evolution of technique of the direct trans-blepharoplasty approach for the correction of lower lid and midfacial aging: Maximizing results and minimizing complications in a 5-year experience. Plast, Reconstr, Surg., 105: 393-406; discussion 407-408, 2000. 